\title{
Design of a Simulation Tool for Audiology Education to provide Hearing Screening Training
}

\author{
John Gerdes \\ Integrated Information Technology \\ University of South Carolina \\ Columbia, SC USA \\ jgerdes@sc.edu
}

\author{
Benjamin Schooley \\ Integrated Information Technology \\ University of South Carolina \\ Columbia, SC USA \\ BSchooley@cec.sc.edu
}

\author{
Dakota Sharp \\ Mongomery Speech-Language-Hearing \\ Clinic \\ University of South Carolina \\ Columbia, SC USA \\ DS24@mailbox.sc.edu
}

\begin{abstract}
Early identification of hearing impairment and ear disorders is important, which is why hearing screening is routinely done on newborns, with regular screening recommended on children through the age of $\mathbf{1 8}$. Screening is also completed with adults to assess and treat hearing problems. Procedural training is needed for new Speech-Language Pathologists and nursing students as well as continuing education for those trained to perform this procedure. An audiology simulator was developed to provide an alternative to traditional face-to-face lab instruction. Using a design science approach, the development of the simulation prototype is discussed. Contributions include a useful framework for developing such a simulation of an existing process, a description of a unique artifact that supports an individualized, self-paced learning environment using context-sensitive feedback and performance assessment, and an extensible approach to supporting virtual subjects in audiological training.
\end{abstract}

Keywords-design science, audiology, simulation, hearing screening, framework

\section{INTRODUCTION}

An audiology simulator was developed to provide an alternative to a traditional face-to-face lab instruction training for Language Speech Pathologists learning how to perform hearing screening assessments. This tool would also be useful for nurses, teachers and audiologists to train on performing this procedure. This paper uses a design science approach to discuss the development of the simulation prototype.

The Hearing Screening test is a fast, inexpensive, and easy to perform pass/refer screening procedure used to identify those who require further audiological evaluation. Those who do not pass a hearing screening (the term fail is not used) is typically referred to a licensed audiologist who would perform more detailed hearing testing.

A hearing screening test can involve three procedures - puretone hearing screening which identifies hearing impairment, otoscopy which is a visual inspection of the outer ear, and tympanometry which measures the movement of the eardrum in response to a change in pressure. Different protocols are used depending on patient's age [1]. For school-age children, hearing screening helps identify those most likely to have a hearing impairment that may interfere with their education, health, development, or communication. Hearing impairment is defined as the inability to hear a pure tone at the $20 \mathrm{~dB}$ HL level [1]. Hearing screening is also completed with adults. Hearing impairment (i.e., loss or abnormality of psychological or physiological function) and/or hearing disability (i.e., restriction or lack of ability to perform an activity, resulting from an impairment) are prevalent chronic conditions among adults of all ages with hearing impairment increasing as a function of age.

Guidelines also exist for screening for outer and middle ear disorders. It is a pass/refer procedure that involves a visual inspection via an otoscope or video otoscope, and acoustic immittance testing via a tympanogram. An otoscopy exam is a clinical procedure used to examine structures of the outer ear, particularly the external auditory canal and tympanic membrane (eardrum). A tympanometry test measures how the eardrum moves and helps diagnose disorders that can lead to hearing loss.

Medical institutions are grappling with how decrease training costs with an increased focus on digital education, including online and distance learning programs, and simulations. Simulation is increasingly being used in medical training. It has been found to improve nursing knowledge and skills, self-confidence, communication skills, empathy, critical thinking abilities, leadership, and situation management among nursing students [2, 3, 3, 4, 6, 7].

Clinical training requires students to gain experience with subjects exhibiting different medical conditions, which is challenging in a training lab. While peers often serve as test subjects for other students, this does not guarantee exposure to, and experience with, the full spectrum of medical conditions.

The American Speech-Language-Hearing Association recognizes the use of standardized patients and simulation technologies as alternatives to clinical education methods [8]. Traditionally, standardized patients (SP) were individuals trained to portray a specific clinical case in a highly consistent and measurable manner [9]. With the introduction of configurable manikins, different SP profiles may be loaded into a test manikin to present students with different scenarios. The National Council of State Boards of Nursing (NCSBN) also supports the use of quality simulations as a substitute for a percentage of clinical hours in undergraduate nursing programs [10]. Generally, little is known about the effectiveness of simulation in audiology education and training $[11,12,13]$. 
Health care professionals need training in many areas, including patient communication with patients, diagnostic procedures, and how to work with appropriate diagnostic equipment. This is true for audiology education. Healthcare professionals need to be familiar with how to operate specialized diagnostic equipment and interpretation of results. However, gaining access to the diagnostic equipment can be difficult. There is a significant expense to equip and staff an instructional lab, which limits the hours the lab is accessible and the number of students that can receive training at any given time.

Hearing Screening tests are administered by those trained to do these tests, including speech audiologists, speech language pathologists, nurses, and teachers [14]. A hearing screening is a quick, inexpensive, easily administered test that results in either a pass / refer result. Passing indicates the patient shows no signs of hearing loss. Not passing means the patient should be referred for a more thorough hearing assessment by a licensed audiologist.

The American Academy of Pediatrics (AAP) recommendations for preventative pediatric health care advocates regular hearing screening for all children from age 4 to 21 years of age, regardless of the presence or absence of risk factors for hearing loss $[15,16]$. This suggests that the 155,000 new nursing graduates each year need training in how to administer audiometric screening tests. In addition, there are over 4 million nurses in the U.S. and 20 million worldwide that would benefit from continuing education training in this area. California, for example, requires registered nurses to complete 30 hours of continuing education contact hours every 2 years to maintain their active license. [17].

Getting the necessary audiology training can be challenging due to capacity limitations of the training lab, or the lack of a convenient facility for remote learners. To address these issues, a hearing screening simulator was explored and created. While simulation is an effective pedagogy for educating health professionals and is used extensively in undergraduate nursing education, it is not in widespread use in the field of audiology training. The authors of this manuscript sought to design and develop a tool that could provide nursing students practice in performing hearing screening tests online, without the requirement to visit the on-campus hearing lab and interacting with live patients. This was particularly important during the COVID-19 pandemic where disease exposure risk was a critical issue. The capacity of the lab was limited in terms of both space and availability of certified trainers making scheduling difficult. Traveling to the physical training lab was also inconvenient for many of the remote students, who live all over North America.

A design science approach was adopted for this project. Initial design specifications were collected for the Hearing Screening Simulator. An iterative process involving the development of a prototype, review, and critique by the lab staff were employed which fed into the next development iteration. After several iterations, the final prototype was tested to assess if students felt the simulator was useful in learning how to administer hearing screening tests and determine students' confidence in their skills after using the simulator.
Contributions of this work include a useful framework for developing such a simulation of an existing process, a unique artifact that supports an individualized, self-paced learning environment using context-sensitive feedback and performance assessment, and an extensible approach to supporting virtual subjects in audiological training.

\section{FOUNDATION AND PRIOR WORK}

A national study found that $14.9 \%$ of children (6-19 years of age) have low- or high-frequency hearing loss in one or both ears [18]. Early identification, diagnosis, and intervention are beneficial, improving the likelihood that children develop effective communication and language skills, and achieve successful learning outcomes [19]. As such, the test must be carried out effectively. This is in part accomplished through adequate training of healthcare students and personnel on how to perform tests and diagnose patients [12].

\section{A. Hearing Screening Test Procedure}

The American Speech-Language-Hearing Association's (ASHA) Guidelines for Audiologic Screening [1] specifies different procedures for doing screening for hearing impairment based on the age of the subject. There are guidelines for screening: newborns and infants age birth through 6 months; infants and toddlers age 7 months through 2 years; preschool children age 3 to 5 years, school-age children age 5 through 18 years; and adults 18 years or older. Of interest to the current study are the guidelines for school-age children ages 3 to 5 ages, children ages 5 to 18 years, and adults 18 years and above. The younger subjects require additional training and different procedures. In part, the guidelines for subjects in the 5 to 18 year age group, specify testing should be done using 1000, 2000, and $4000 \mathrm{~Hz}$ tones at $20 \mathrm{~dB} \mathrm{HL}$, and that the test is repeated at least twice at each frequency to assure reliability. For adults, the testing should also be done using 1000, 2000, and $4000 \mathrm{~Hz}$ tones, but with a $25 \mathrm{~dB}$ HL setting.

ASHA's Guidelines for Audiologic Screening [1] also provides guidelines for screening the outer and middle ears. This procedure, which is the same for all age groups, includes an optional case history, visual examination (otoscope examination), and acoustic immittance testing (tympanogram).

\section{B. Prior Work}

Studies have shown simulation to be effective in audiology education and training $[21,22,23]$. Simulation can help transfer theory to practice in an integrated teaching and learning model [24]. Simulators can present a full range of patient cases that represent real-world clinical diagnoses. These simulated cases present patients with symptoms that must be detected and diagnosed by the student. However, the use of simulation in audiology education is still in its infancy [12].

The authors are not aware of any existing simulation tools that model the operation of commercial audiometry devices, or that provide the means for students to train on the use of such devices. CARL, sold by AHead Simulations ${ }^{1}$ is a physiologically and acoustically realistic desktop manikin that enables clinicians and students to easily and safely train and

\footnotetext{
${ }^{1}$ https://www.aheadsimulations.com/
} 
develop their skills. "CARL is so realistic that you can see inside his ear, make measurements, fit hearing aids and tubes, AND generate accurate readings that indicate how well the hearing aid is fitted" 2 . Some of the applications that have used CARL include an introduction to audiometry and occupational hearing, serving as a standardized patient for audiometry and the fitting of hearing aid training, evaluating hearing aid streaming of music and speech, and the programming and verifying telecoil programs. It is suitable only for in-person training using commercial clinical test equipment since CARL simulates standardized patients, not clinical instruments. While CARL is a useful educational tool for audiologists who will go on to provide diagnostic evaluations and hearing aid programming, the technology is too complex and not suited for training providers who will go on to complete hearing screenings.

Prior work in audiology simulation education includes the use of an immersive training system that was found to provide audiology students with better learning outcomes and selfconfidence than found with traditional training [7]. Simulated training has been found to help with student's self-confidence in performing audiology testing [7, 21]. The use of standardized patients for training on infant hearing screening and parental counseling simulation was found to improve clinical skills and increase confidence levels of audiology students [21]. In one study, significant improvements in clinical skill levels and higher confidence were observed when students completed the simulation task of preparing an adult mannequin for an auditory brainstem response (ABR) test [13]. In another study, the incorporation of simulation workshops in Speech and Language Therapy curricula was found to have a positive quantitative and qualitative impact on students' perception of learning [25]. The critical review and debriefing of students by qualified practitioners following simulated training is an important aspect of an experiential learning experience. [26].

\section{RESEARCH APPROACH}

This research follows a design science research (DSR) methodology consisting of (i) problem identification and motivation, (ii) definition of the objectives for a solution, (iii) design and development, (iv) demonstration, (v) evaluation, and (vi) communication [27, 28]. We identified and translated design requirements for an audiology simulator into design components. We then classified these functional components in the app into design principles and features for designing a healthcare simulation app for clinical education and training. We conform our meta-artifacts to mode $3 \mathrm{C}$ and $4 \mathrm{~B}$ of design theorizing, i.e. seek to contribute to knowledge for solution design process and system to inform the design of the DSR project system and codify effective design principles and features [29] that contribute to future DSR projects of similar context. The design requirements, artifact design and development, and evaluation are described below.

\section{DESIGN REQUIREMENTS}

Hearing clinic personnel sought a tool to train students in an online Master's of Speech Language Pathology program on how

\footnotetext{
2 https://www.acceleratorcentre.com/news/although-he-maylook-like-one-carl-is-no-dummy
}

to administer hearing screening assessments. This involves performing three separate tests - otoscopy which takes a picture of the outer ear, tympanometry which measures the movement of the eardrum in response to a pressure change, and a pure-tone audiometer which measures hearing impairment. The clinic indicated that no such simulator existed, and the development would be useful, making it easier for students and practitioners to more easily train in performing these assessments. The goal of the research was to develop an effective simulator that could replicate as close as possible the experience of administering and interpreting a hearing screening test. To guide this research, a conceptual model of the application was first developed.

\section{A. Conceptual Framework}

Before an effective solution could be developed, it is necessary to understand the problem. Since the goal was to simulate an existing process, it was necessary to understand all the elements that play a role in that process and understand how they interact. To simulate a hearing screening test there are two actors, namely the technician administering the test, and the subject being tested. Also critical is the testing environment which includes the clinical equipment needed to perform the test. A less obvious element relates to systems that might address administrative requirements. These might be administrative or reporting functions that are done in the background. Developing an understanding of how actors interact is also critical. An effective approach is to develop separate Use Cases to detail how actors interact and what information is being transferred. For the current project, there are five Use Cases, namely: authenticating and authorizing the technician, collecting patient history, following the proper protocol and performing the hearing screening, recording of technician notes during the examination, and assessing the accuracy of technician's examination process and subsequent results.

\section{B. Developing Design Requirements}

Design requirements were gathered in four iterations with a clinical assistant professor at the University of South Carolina Montgomery Speech-Language-Hearing clinic in the Arnold School of Public Health. A series of informal interviews were conducted to gather and clarify requirements at the outset of the project as well as during software development. An agile development model was used. Once an operational prototype was developed, the clinical instructor would evaluate it and provide feedback, which initiated the next round in the development. System specifications did expand as the project progressed. The initial object was to have a simulator that would give students exposure to the audiometer but automatically assess student performance. As the capabilities of the simulator increased, so too did the objectives of the project.

Meta-requirements fall into two categories - clinical requirements necessary for performing a hearing screening evaluation, and those included for operational reasons. The clinical requirements reflect the data that would be collected and presented using a traditional training session with commercial equipment. The operational requirements reflect additional features that exceed the clinical requirements and enable a web- 
based digital learning experience, such as calculate a student grade and therefore represent value-added aspects of the simulator. The Meta requirements included:

\section{1) Clinical Requirements}

- Authenticate and authorize a user.

- $\quad$ Graphical user interface (GUI) that has a similar look and feel to standard audiology testing hardware-based systems

- Ability to simulate three audiometry tests - video otoscopy, tympanometry, and audiometry

- Audiometer testing only needed for a specific subset of frequencies used for hearing screening

- Display output similar to that obtained with commercial devices, including otoscope images, tympanograms, and audiometer charts.

- Include standardized patients representative of the range and scope of real-world clinical cases

2) Operational Requirements

- Web-based platform

- $\quad$ Tracking and reporting of time spent on the simulation

- Ability to document and printout a record of the simulation session

- $\quad$ Provide automatic grading and feedback of student's performance

- Provide a full-featured audiometer that provides features beyond those needed for the hearing screening learning experience, but is present in commercial units.

- Log all simulation testing to permit reporting of an individual student's testing history, and enable crosssectional data analysis across multiple students' results

A simulation objective was to get students familiar with the audiology equipment and comfortable administering hearing screening tests. Having a similar look and feel to commercial equipment was, a high priority. To complete a hearing screening test, students need to be familiar with all three required testing modes (i.e., otoscope, tympanometer, and audiometer), so inclusion was important. Since the focus was on screening, and not a full hearing assessment, the system only had to simulate a limited set of test points. Being able to interpret test data and reach a correct result was also a key goal, and so it was important to present the results in an industry-standard format. The initial requirements did not include standard test subjects. This requirement was added in an early prototype review. We started with a single subject, which was eventually expanded to ten.

Of the operational requirements, porting the simulation remains a high priority. The Windows application is stable and performing as expected. With this goal of moving the simulator to the web, the inclusion of extra Metadata that reports time spent and the number of tests completed will help give training supervisors confidence that the student has completed the training. Being able to print out the session record lets the students document their performance. Automatic grading provides the learner immediate feedback and reinforces the learning process. Having a full-featured simulator allows the curious learner to explore on their own, and also allows the simulator to be used for training other than for just hearing screening (i.e., completing more comprehensive audiology testing). Also having the ability to assess results across multiple student technicians may help identify weaknesses and strengths in the classroom training.

\section{ARtifact Design And DEVELOPMENT}

The Hearing Screening Simulator (HSS) was developed as a Windows form application using Visual Basic as the development language. Visual Basic was used because it supported a graphical user interface, integrated charting libraries needed for the audiology graphs, and the ease with which it could be ported to a web-based platform in the future. The design of the simulator unit was based on the look of existing audiology testing units in the lab. This was done to acquaint students with the layout and operation of these devices and to provide a more realistic simulation experience.

\section{A. Addressing Clinical Requirements}

The HSS is designed to simulate three clinical tests used in earing screening and audiological evaluations. An otoscope exam helps to assess the condition of the external auditory canal and tympanic membrane. This is a visual process. A doctor may use a traditional otoscope to look into the patient's ears, or, as in our simulation, use a camera to capture an image. Tympanometry tests how well the subject's eardrum moves, as well as determine the volume of the ear canal and pressure of the middle ear space. The audiologist will put a small probe, which looks like an earphone, into each ear. A small device attached to the probe will push air into the subject's ear, and the device measures the resulting response. This creates a graph, which is displayed on the test unit [14]. The audiometer is used to determine the quietest sound the subject can hear at different pitches or frequencies. A tone is presented and the subject indicates if they heard it by raising their hand. In a screening, however, tones are presented at a fixed level considered "normal hearing” (20 dBHL), rather than determining the softest level the subject can hear.

All student interaction with the simulator is stored in a SQL database. This also handles user authentication and authorization. The simulator user interface consisting of three panels is shown in Fig. 1. The left panel presents the simulated audiometer device. Similar to commercial audiometers designs, the upper portion of the unit contains a graphic display that shows either the tympanogram or audiology chart depending on the testing mode selected. The lower portion of the unit provides the various controls used to perform the testing. These controls and their purpose are described below. The Center section provides information about the subject, and command buttons to display and print a larger version of the audiogram chart, and to display the test debriefing report which summarizes the student performance on the simulation. The right panel is for Technician Notes. It allows the student to record their findings and include observation as is typical in a traditional audiological screening.

The user interface is designed with interactive objects (see Fig. 1). As buttons are pressed the simulator provides sensory 


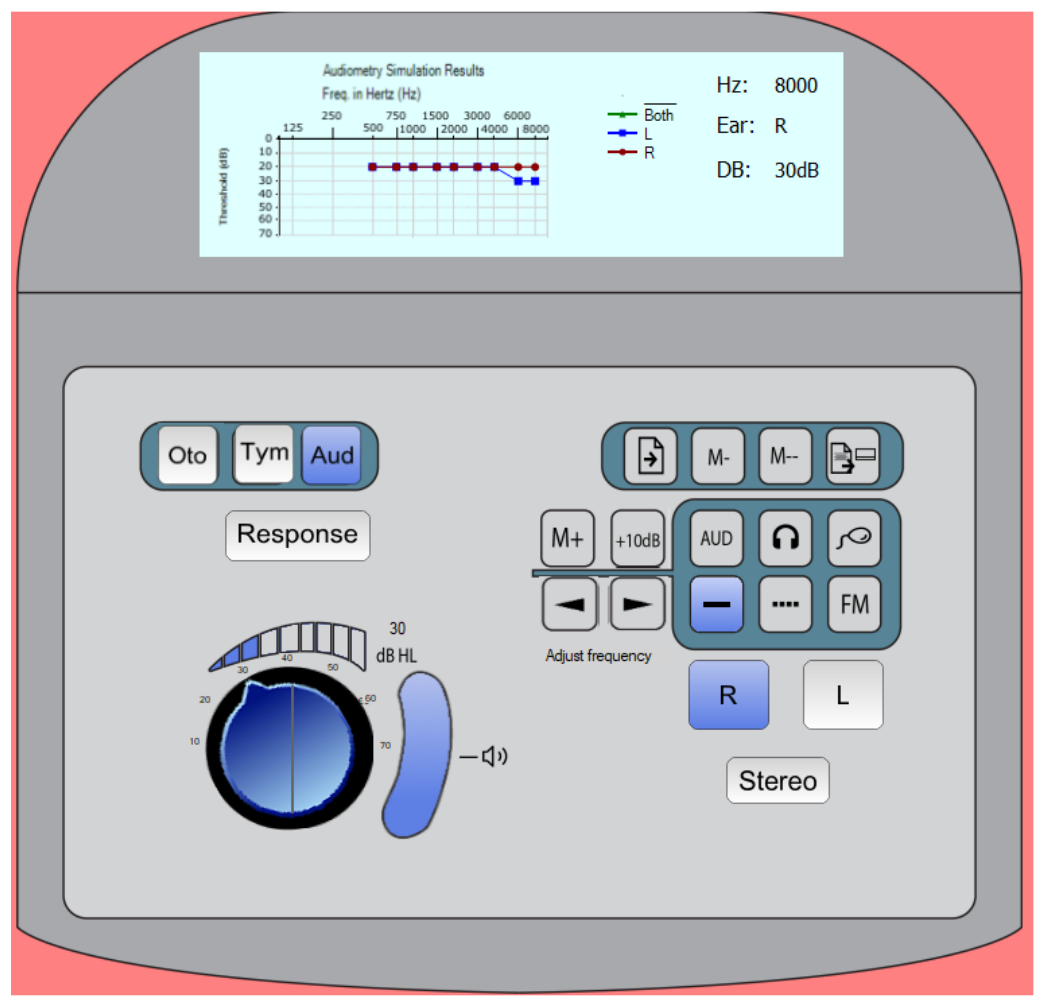

A. Simulated Audiometer

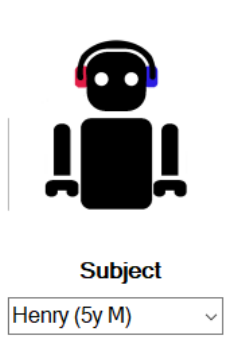

Henry's father reported that Henry has a history of ear infections requiring surgery. He noted no recent hearing concerns

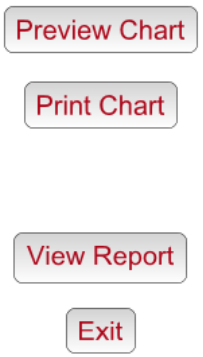

B. Subject Window

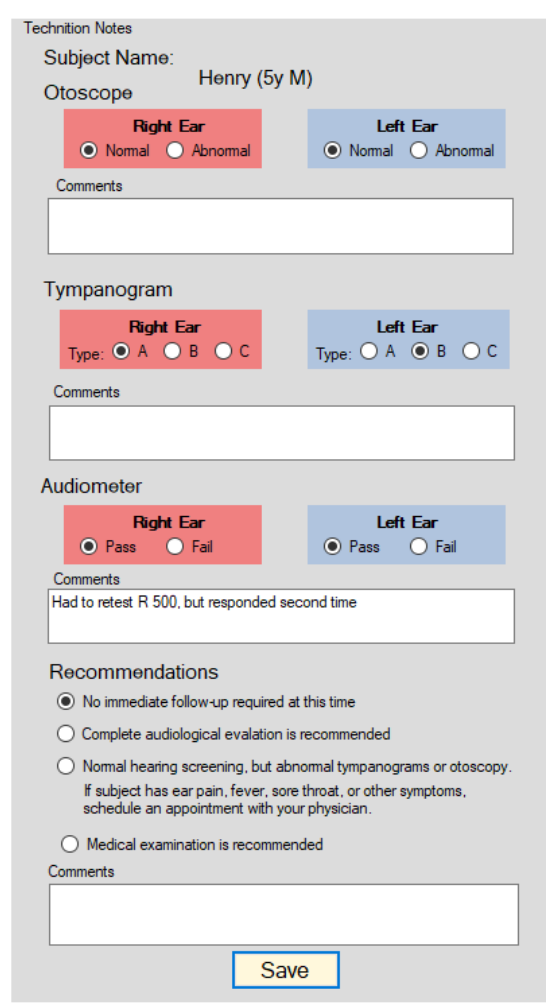

C. Technician Notes

Fig. 1: Hearing Screening Simulator. The left panel simulates the operations of the audiometer, the center panel gives subject details with the avatar responding to tone stimulus, and the right panel providing an area for technician's result and comments.

feedback to let the user know the action was registered. For example, when the user selects one of the three buttons in the top left, it selects the testing mode and toggles the button color (blue indicates that option is selected). This is accomplished by loading either the selected or unselected image into the object when a button is pressed. The audiology chart was created using the native charting capability of Visual Basic.

The simulator's three testing modes are 'Oto' to display an otoscope image, 'Tym' to display the tympanogram of the target ear, or 'Aud' to perform an audiological test. The bank of buttons on the right control the test conditions for the audiometer. This includes frequency in $\mathrm{Hz}$, intensity level in $\mathrm{dBHL}$, sample type (being either pure tone, pulsed, or frequency modulated), and controls to specify which ear is to be tested (right, left, or both (stereo). The large dial on the bottom left provides an alternate way of controlling the intensity level presented to the subject. The intensity level is graphically displayed in the progress bar above the dial.

Modeled on the design and operation of commercial devices, the curved button is referred to as the 'Present' button, which serves one of two functions based on the testing mode. On commercial devices a tympanogram test begins automatically when a seal is obtained on the ear canal. Since the simulator did not model acquiring this seal, there needed to be a way to initiate the test. The present button was used to trigger the test and displays the resulting tympanogram chart. There is no operator or subject response needed for this test - the test is fast and automatic. The HSS displaying a stored image file representing the subject's tympanogram chart after the student presses the present button. it is meant to simulate a tympanogram test. When the user presses the curved Present button in the 'Aud' (audiometer mode) the test tone is presented to the subject. To provide visual confirmation that the tone was played, an indicator light is displayed next to the avatar. Note, this is meant as an indicator to the technician, and no such indicator would be visible to the subject in real audiological tests.

The subject avatar raises its hand if the simulation 'hears' the presented tone (Fig. 2). If the avatar raises their hand, the technician presses the Response button indicating the subject responded to the tone. HSS records that the subject responded to the signal at the specific frequency and decibel level. If there is no response, the tone can be replayed, or the decibel level increased until the subject does give a response. The test process frequency can then be indexed to the next test point and the process repeated. This process records the subject's response on the audiogram at the top of the simulator. Data for the right, left and both ears (stereo) are included on the same chart. The technician has the option to enlarge and print the diagram using the controls in the center panel.

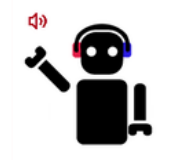

Fig. 2: Avatar responding to a tone stimulus in the right ear. Note, speaker icon is displayed to indicate to the technician that the tone was presented in the event the local speakers are not enabled. 
In the audiogram mode, when a tone is presented to the subject, a representative tone is also played for the technician (i.e., the student) and an indicator light illuminated (see Fig. 2). The frequency and intensity of the tone played through the computer's speakers are also modulated to simulate what the subject is hearing. To model the operation of actual audiology devices, the simulator can perform testing using constant tones, pulsed tones, or frequency modulated samples. Testing can be done on the right or left ear, or the stereo setting can be used to present the tones in both ears simultaneously. When the simulator is in the 'Tym' (tympanogram) mode, the tympanogram image of the target ear is displayed in the graphic display at the top of the unit. There are controls to change the testing frequency between nine specific values between $500 \mathrm{~Hz}$ and $8000 \mathrm{~Hz}$. Some controls allow the operator to modulate the sound intensity level from $10 \mathrm{dBHL}$ to $70 \mathrm{dBHL}$.

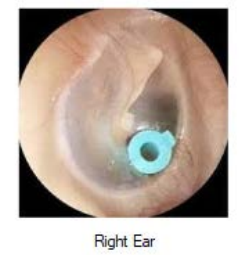

A. Otoscope Image of the right ear

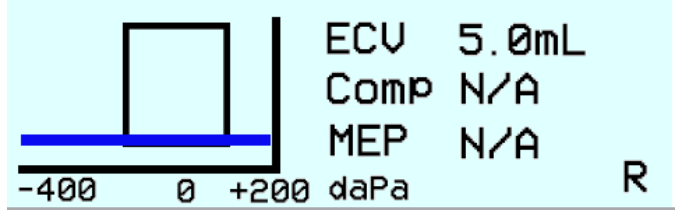

B. Tympanogram trace for the right ear

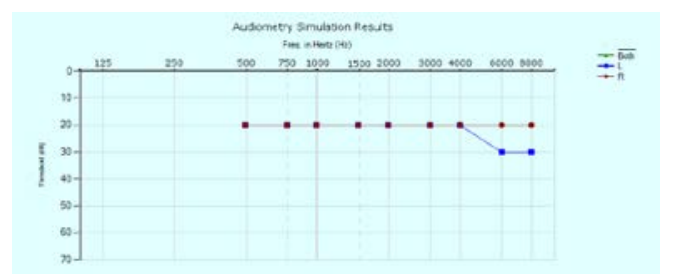

C. Audiogram for the right and left ears.

Fig. 3: Simulator otoscope, tympanogram, and audiogram outputs generated by the Hearing Screening Simulator.

A sample otoscope image, tympanogram, and audiogram chart are shown in Fig. 3. Note, the otoscope images and tympanograms are specific to the subject's ear being tested. Those images are part of the subject profile that is loaded when the subject is selected. In contrast, the audiogram is dynamically created based on the technician's recording of the subject's responses. The chart shows data from both ears on the single graph. Note, the audiogram shows subject responses at more frequencies and intensity levels than used for screening tests.

\section{B. Addressing Operational Requirements}

The HSS simulator is equipped with 10 standard patients, although the set of standard patients could also be expanded to enhance the catalog of clinical cases covered by the simulator. Each patient has a short backstory which is presented to the student when the subject is selected. This provides the age and gender of the subject, and a brief background comment related to any hearing concerns. The ten subjects were created with varying audiologic conditions and pathologies, with audiometry, tympanometry, and otoscopy results consistent with their condition. One child and adult standard patient with normal results were included as well. To help mask the identity of these standard patients and give the illusion of having a larger subject base, the profile information is randomized each time the program is run. The genders are changed, age shifted slightly, the name of the subject being selected from a large list of diverse names, and the backstory adjusted to match the new subject profile. This not only prevents someone from circumventing the training by utilizing results from earlier tests, but it also enables repetitive testing on the same standardized subject without it being evident it is the same subject.

As the student goes through the simulation, they are asked to record their observations and patient results in the Technician Notes panel. There are note sections for each of the three tests (otoscope, tympanogram, and audiometer). The student is asked to provide a result for each ear and to add comments and observations related to that test. Having the student enter this information into the simulator allows the simulator to assess their performance. The system knows which result is correct based on the standard subject being tested. Taking into consideration the three individual tests, the student must also make a recommendation regarding the patient's subsequent follow-up, and also has the opportunity to add a related comment. This final recommendation can also be automatically graded by the simulator and provide appropriate feedback as needed. The patient cases, associated correct result, and correct recommendations were defined by the project clinical team and implemented in the application database.

Once the student completes the testing and enters their session notes, the data is saved to a database and a session report is generated (see Fig. 4). This report serves as a debriefing for the simulated training. It provides Metadata related to the testing, including the technician's name (i.e., the student's name), the subject's name, the duration of testing, which tests were run during the evaluation, as well as the total time spent using the hearing screening application. This session report provides information to the training supervisor to show what steps the student went through during the session. If the student took shortcuts during the session, this data would make that evident. The report also shows the student's results along with the correct results coupled with an explanation justifying the result. This gives the student immediate feedback on their performance. These reports can be printed out or saved as a pdf.

\section{EVALUATION}

Thirty-three first year speech-language pathology graduate students enrolled in an introduction to audiology class participated in this study. All participants received the same standardized lecture covering concepts surrounding hearing loss and how to perform hearing screening assessments. Students were randomly assigned to the control $(n=16$, female $=16)$ or test $(n=17$, female $=17)$ groups. The control group received brief in-person training to explain how to operate the equipment, and then were evaluated conducting their live, in-person 
Simulation Summary

Technician: John Smith

Date: $\quad$ Wednesday, June 2, 2021 9:34:46 AM

\begin{tabular}{|l|l|l|l|}
\hline Testing duration: & \multicolumn{3}{|c|}{$36.6 \mathrm{Min}$} \\
\hline Number Of Subjects tested: & \multicolumn{3}{|c|}{1} \\
\hline Auditory tests run: & 21 right & 4 left & 0 both \\
\hline Tympanometry tests run: & 1 right & 1 left & \\
\hline Otoscope images viewed: & 1 right & 1 left & \\
\hline
\end{tabular}

\begin{tabular}{|c|c|c|c|}
\hline \multirow[t]{2}{*}{ Subject: } & \multicolumn{3}{|c|}{ Henry (5y M) } \\
\hline & \multicolumn{3}{|c|}{$\begin{array}{l}\text { Henry's father reported that Henry has a history of ear } \\
\text { infections requiring surgery. He noted no recent hearing } \\
\text { concerns. }\end{array}$} \\
\hline Otoscope & Right & \multicolumn{2}{|c|}{ Left } \\
\hline Status: & {$[x$ - incorrect $]$} & Normal & [X- Incorrect] \\
\hline \multicolumn{4}{|l|}{ Comment: } \\
\hline $\begin{array}{l}\text { Correct } \\
\text { Response: }\end{array}$ & $\begin{array}{l}\text { Abnormal, pressure equalization tube present in } \\
\text { eardrum }\end{array}$ & Abnorma & \\
\hline Tympanogram & Right & & \\
\hline Type: & {$[X$ - Incorrect $]$} & Type B & [v-Correct] \\
\hline \multicolumn{4}{|l|}{ Comment. } \\
\hline $\begin{array}{l}\text { Correct } \\
\text { Response: }\end{array}$ & $\begin{array}{l}\text { Type B, no eardrum movement and large ear canal } \\
\text { volume }\end{array}$ & $\begin{array}{l}\begin{array}{l}\text { Type B, n } \\
\text { volume }\end{array} \\
\text { nol }\end{array}$ & nent and large ear canal \\
\hline Audiometer & Right & & \\
\hline Screening: & {$[\checkmark$ - Correcl] } & Pass & {$[v$-Correct $]$} \\
\hline Comment: & \multicolumn{3}{|l|}{ Had to retest R 500 , but responded second time } \\
\hline $\begin{array}{l}\text { Correct } \\
\text { Response: }\end{array}$ & Pass & Pass & \\
\hline \multicolumn{3}{|c|}{ Recommendation: No immediate follow-up required at this time } & {$[x$ - incorrect $]$} \\
\hline \multicolumn{4}{|l|}{ Comment: } \\
\hline $\begin{array}{l}\text { Correct } \\
\text { Response: }\end{array}$ & \multicolumn{3}{|c|}{$\begin{array}{l}\text { Normal nearng screening. but abnormal tympanograms or otoscopy. If you have ear pain. fever. sore } \\
\text { throat, or other symptoms, schedule an appointment with your physician }\end{array}$} \\
\hline
\end{tabular}

Fig. 4: Simulation Report including session Metadata and Simulation Debriefing Report detailing student result and notes, along with the correct result for the subject.

audiology test. The test group received the same in-person equipment introduction and used the simulation tool before being evaluated doing their live audiology test. Students in each group were divided into two-member teams, with one group of three in the test group. Each student team rotated between roles, acting as a test subject (i.e., patient) and acting as the test evaluator. Approximately half of the students in each group had previous hands-on experience with a hearing screening audiometer. Students were sorted into an approximately equal number of pairings of "no previous experience," "one partner with previous experience", and "both partners with previous experience".

Participants in the test group received supplementary training with the simulation tool. They were introduced to the simulator and were allowed to work with it for about 15 minutes, working through approximately five cases with their partner. A 25 item questionnaire was administered to students to assess their confidence in their skills both prior to and after performing the live hearing screening test. The questionnaire was created by the audiology staff to gauge students' knowledge and confidence in administering and interpreting hearing screening tests. A five-point Likert scale was used. Response options and subsequent coding values were Strongly Disagree (1), Disagree (2), Neither Disagree or Agree (3), Agree (4), and Strongly Agree (5). A set of seven to nine questions were asked related to each of the assessment modes (otoscope, tympanogram, and audiometer). The test group was also asked four additional free response questions in the same questionnaire to assess perceptions about the simulation tool. A total of 16 surveys were completed by each group. One survey was not usable and was thus removed from the analysis.

\section{A. Student Hearing Screening Assessment}

A live assessment of student clinical technique was completed using commercial audiometry units using peers as test subjects. Supervising certified instructors observed the tests and graded the student's performance and technique.

It was expected that using the simulation training tool together with in-person training would be perceived by students to be at least as effective, and possibly an improvement, over inperson only training using commercial audiology equipment. Though some indication of improvement was expected, statistically significant differences between groups were not expected for most questionnaire items between the test and control groups. All statistical analyses were conducted using a standard, commercially available statistical software tool (NCSS 2019 Statistical Software). Thematic analysis of qualitative responses was conducted to produce categorical findings and related discussion.

\section{RESUlTS}

\section{A. Instructor Evaluation of Student Technique}

Supervising certified instructors observed the student testing and graded the student's performance and technique. All students in both the control and test group performed the three tests satisfactorily (i.e., otoscopy, tympanogram, hearing screening). Some students, primarily in the control group, did not follow the prescribed protocol exactly, as reflected in the technique scores shown in Table 1. Students performed similarly across test and control groups except in the case of providing instructions and testing according to protocol.

\section{B. Survey Results}

The test group resulted in a higher post-test mean score and a higher percent change than the control group for all three test component groupings: Otoscope, Tympanogram, and Hearing Screening (see Table 2). To test for statistical significance between groups, a non-parametric analysis was conducted using the Kruskal-Wallis one-way ANOVA to test differences between the change in test and control group pre-test vs posttest survey responses (post-test mean score minus pre-test mean score). The test was corrected for tied ranks.

TABLE 1. TECHNIQUE ASSESSMENT BY CERTIFIED TECHNICIANS IN LIVE TESTING USING COMMERCIAL AUDIOMETRY EQUIPMENT

\begin{tabular}{|l|c|c|}
\hline & $\begin{array}{c}\text { Control Group } \\
(\mathbf{n}=\mathbf{1 6})\end{array}$ & $\begin{array}{c}\text { Test Group } \\
(\mathbf{n}=\mathbf{1 6})\end{array}$ \\
\hline Otoscope & 4.73 & 4.47 \\
\hline Tympanogram & 4.67 & 4.35 \\
\hline Audiometry & & \\
\hline Provide Instructions & 2.53 & 4.50 \\
\hline Test according to protocol & 3.67 & 4.76 \\
\hline
\end{tabular}


TABLE 2. MULTI-FACTOR ASSESSMENT OF STUDENT CONFIDENCE CHANGE. PRE-TEST AND POST-TEST VALUES REPRESENT THE AVERAGE FROM BOTH CONTROL AND TEST GROUPS.

\begin{tabular}{|c|c|c|c|c|c|c|}
\hline & \multicolumn{2}{|c|}{ Control Group (n=16) } & \multicolumn{3}{c|}{ Test Group (n=16) } \\
\hline & $\begin{array}{c}\text { Pre } \\
\text { Test }\end{array}$ & $\begin{array}{c}\text { Post } \\
\text { Test }\end{array}$ & $\begin{array}{c}\text { Change } \\
\text { (\% Change) }\end{array}$ & $\begin{array}{c}\text { Pre } \\
\text { Test }\end{array}$ & $\begin{array}{c}\text { Post } \\
\text { Test }\end{array}$ & $\begin{array}{c}\text { Change } \\
\text { (\% Change) }\end{array}$ \\
\hline $\begin{array}{c}\text { Otoscope } \\
\text { (7 factors) }\end{array}$ & 3.59 & 3.87 & $\begin{array}{c}0.28 \\
(7.8 \%)\end{array}$ & 3.77 & 4.35 & $\begin{array}{c}0.51 \\
(15.3 \%)\end{array}$ \\
\hline $\begin{array}{c}\text { Tympanogram } \\
\text { (9 factors) }\end{array}$ & 3.60 & 4.25 & $\begin{array}{c}0.64 \\
(17.9 \%)\end{array}$ & 3.85 & 4.66 & $\begin{array}{c}0.77 \\
(21.2 \%)\end{array}$ \\
\hline $\begin{array}{c}\text { Hearing } \\
\text { Screening } \\
\text { (9 factors) }\end{array}$ & 3.90 & 4.32 & $\begin{array}{c}0.42 \\
(10.6 \%)\end{array}$ & 3.85 & 4.85 & $\begin{array}{c}0.64 \\
(17.9 \%)\end{array}$ \\
\hline
\end{tabular}

As shown in Table 3, three question responses showed statistically significant differences between groups, including: "I am confident in my ability to explain hearing screening procedures to a child (Chi square $=5.27, \mathrm{p}=.02, \mathrm{df}=1)$; " "I am confident in my ability to determine if otoscopy is normal" (Chi square $=5.14, \mathrm{p}=0.02, \mathrm{df}=1$ ); and "I am confident in my ability to determine if otoscopy is abnormal" (Chi square $=4.86$, $\mathrm{p}=.03, \mathrm{df}=1$ ). Results were not significant across 22 survey questions at the $\mathrm{p}=.05$ significance level and thus showed no statistically significant difference attributable to the use of the simulator.

\section{Free-Response Question Results}

The test group was asked four free response questions regarding their impression of the simulator tool. The first asked “what aspects of the simulation did you like?” The responses were grouped into common themes, namely:

- Provides hands-on experience, helped me become more familiar with an audiometer \& increased my confidence (5 responses)

- $\quad$ Ease of use (4 responses)

- Practice with multiple virtual patients (4 responses)

- Provided useful feedback on the test debriefing report (1 response)

- Thought it was helpful (1 response)

The responses indicate that the students found the hands-on experience provided by the simulator provided an easy to use and useful learning experience with the audiometer that increased the students' confidence in their ability to perform hearing screening testing.

Second free response question asked "what aspects of the simulator did you not like?” This also elicited five thematic responses, namely:

- Did not show how to do otoscopic or tympanometry exam (4 responses)

- Having better instructions would be helpful (4 responses)

- $\quad$ Nothing, this is a useful simulation (2 responses)

TABLE 3. INDIVIDUAL SURVEY ITEM ANALYSIS GROUPED BY OTOSCOPY, TYMPANOGRAM, AND HEARING SCREENING QUESTIONS. KRUSKAL WALLACE ONE-WAY ANOVA CONDUCTED FOR STATISTICAL SigNIFICANCE

\begin{tabular}{|c|c|c|c|c|c|c|}
\hline & & \multicolumn{2}{|c|}{ Control } & \multicolumn{2}{|c|}{ Test Group } & \multirow[b]{2}{*}{$P=.05$} \\
\hline & & Pre & Post & Pre & Post & \\
\hline \multirow{7}{*}{ 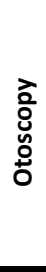 } & I am confident in my ability to interpret otoscopy. & 3.63 & 3.88 & 3.75 & 4.13 & 0.74 \\
\hline & I am confident in my ability to complete otoscopy on a patient. & 3.18 & 3.75 & 3.31 & 4.25 & 0.39 \\
\hline & I am confident in my ability to locate landmarks while completing otoscopy. & 3.31 & 3.69 & 3.63 & 4.44 & 0.08 \\
\hline & I am confident in my ability to determine if otoscopy is abnormal. & 3.81 & 3.60 & 3.81 & 4.25 & $0.03^{*}$ \\
\hline & I am confident in my ability to determine if otoscopy is normal. & 3.94 & 4.00 & 3.94 & 4.50 & $0.02^{*}$ \\
\hline & I am confident in my ability to determine if otoscopy warrants a referral. & 3.5 & 3.94 & 3.88 & 4.25 & 0.43 \\
\hline & I am confident in my understanding of the impact of abnormal otoscopy on hearing screening results. & 3.81 & 4.25 & 4.06 & 4.60 & 0.32 \\
\hline \multirow{9}{*}{ 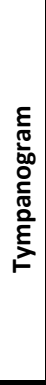 } & I am confident in my ability to interpret tympanogram. & 3.5 & 4.40 & 3.85 & 4.69 & 0.43 \\
\hline & I can confidently identify a normal ear canal volume (ECV) on a tympanogram. & 3.81 & 4.31 & 4.06 & 4.69 & 0.45 \\
\hline & I can confidently differentiate between tympanogram types. & 3.63 & 4.31 & 4.00 & 4.69 & 0.76 \\
\hline & I can confidently identify an abnormal tympanogram & 3.69 & 4.31 & 4.13 & 4.75 & 0.66 \\
\hline & I can confidently identify a normal tympanogram. & 3.88 & 4.38 & 4.25 & 4.81 & 0.86 \\
\hline & I can confidently complete a tympanogram on a patient. & 3.06 & 4.27 & 3.25 & 4.63 & 0.51 \\
\hline & I am confident in my ability to determine if a tympanometry result warrants a referral. & 3.69 & 4.13 & 3.63 & 4.56 & 0.12 \\
\hline & $\begin{array}{l}\text { I am confident in my understanding of the impact of abnormal tympanometry on hearing screening } \\
\text { results. }\end{array}$ & 3.75 & 4.19 & 3.75 & 4.63 & 0.85 \\
\hline & I am confident in my ability to connect abnormal otoscopy results to abnormal tympanometry results. & 3.43 & 3.94 & 3.69 & 4.50 & 0.76 \\
\hline \multirow{8}{*}{ 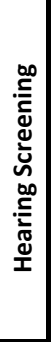 } & I am confident in which frequencies should be tested during a hearing screening. & 4.31 & 4.73 & 4.13 & 4.69 & 0.24 \\
\hline & I am confident which dB level should be tested during a hearing screening. & 4.13 & 4.50 & 3.75 & 4.56 & 0.07 \\
\hline & I am confident in my ability to explain hearing screening procedures to an adult. & 3.5 & 4.25 & 3.75 & 4.69 & 0.42 \\
\hline & $\begin{array}{l}\text { I am confident in my ability to explain hearing screening procedures to a child (e.g., conditioned play } \\
\text { audiometry). }\end{array}$ & 3.63 & 3.93 & 3.47 & 4.38 & $0.02 *$ \\
\hline & I am confident in how to present stimuli/tones while completing a hearing screening. & 3.75 & 4.33 & 3.56 & 4.69 & 0.11 \\
\hline & I am confident in how to verify a response while completing a hearing screening. & 4.00 & 4.25 & 4.00 & 4.56 & 0.22 \\
\hline & I am confident in knowing when a referral is necessary based on hearing screening results. & 4.00 & 4.50 & 3.93 & 4.31 & 0.60 \\
\hline & I am confident in knowing which professional to refer to based on hearing screening results. & 4.38 & 4.44 & 4.38 & 4.47 & 0.75 \\
\hline
\end{tabular}


- $\quad$ It was repetitive (1 response)

- $\quad$ Did not look like the audiogram machine (1 response)

The simulator was designed to display an image or chart for a virtual subject and not how to perform an otoscopic or tympanometry exam. Students wanted more explanation as to how to perform these exams. Others felt the instructions, in general, could be improved, possibly incorporating pictures or a video. One comment expressed a concern that the design of the simulator did not look like the audiogram machine. Given the simulator was modeled to look similar to the GSI 39 Combination Audiometer and Tympanometry device, it is assumed the student was familiar with a different model.

The third question asked "do you have any recommendations to improve the simulator?” Response themes were:

- The simulator did not show how to perform otoscope and tympanometry. Clearer instructions are needed in general (5 responses)

- $\quad$ Nothing, no improvement needed. (3 responses)

- Model the technician giving required directions to the subject (1 response)

- Have the design of the simulator match that of the audiometer (1 response)

The most frequent response was that the simulator should improve training on how to perform otoscopy and tympanometry testing. It was also suggested that the simulator model the instructions provided by the testing technician to the subject. Future software iterations may consider these changes.

The fourth question asked the student "would you like to share anything else?” Responses included:

- “A great tool to add to any audiology class” (1 response)

- "Really liked that sample participants showed inconsistencies in hearing thresholds. This realistic touch prepares one to be not thrown off when performing a live, in-person test.” (1 response)

- “The simulation was easy to use” (1 response)

- “The virtual simulation was very helpful” (1 response)

- “I loved the activity!” (1 response)

Responses to the final question noted positivity towards the simulation tool indicating its potential benefit for providing a range of realistic learning experiences. It does suggest however that some feature of the simulator need to be discussed further in the system documentation. The final three comments reinforced that the simulator was easy to use, and perceived as a useful addition to the class.

\section{DISCUSSION}

Our HSS is a novel solution that may be useful in training future Speech-Language Pathologists, student nurses, and other professionals in the performance of hearing screening testing. This is the only known solution that permits learners to perform a hearing screening assessment on simulated patients, and receive constructive feedback on their performance. It addresses a need for remote training that cannot be provided using commercial audiology equipment, and thus represents an improvement [27] on the state-of-the-art. It provides a heretofore unavailable avenue for experiential learning for online and remote learners. The design science research framework provided in this study may be useful for developing simulation training, generally, and audiology simulation training specifically. The software simulation tool provides a design artifact that was tested and evaluated across 32 participants and thus provides a complete DSR cycle inclusive of artifact evaluation.

This study provides a comprehensive technical investigation and evaluation of the requirements for the simulator focused on the user interface design, and user experience influencing the system design artifact instantiation of an HSS. In the evaluation, we surveyed users to assess usability, and efficacy as a teaching tool. While the mean scores for the test group were largely higher than the control group and demonstrated larger percent change from pre-test to post-test, there were few statistically significant differences between groups. This was not surprising considering the very early phase of research in this study and the known difficulties measuring educational interventions especially with small sample sizes. Taken together, the quantitative and qualitative results from this study show significant promise for audiology simulator tools for enhancing audiology education. The results of this study indicate the working prototype of the simulator may provide a strong supplement to in-person training using commercially available equipment. Participants noted the simulator was intuitive and useful for learning the skills needed to perform hearing screening tests while also indicating areas for improvement.

\section{A. Limitations}

The number of participants is low $(n=33)$ yet appropriate for an initial pilot test. Future studies should include a broader range of participants. This study represents the first test of the simulation software. Future iterations of the software with prescribed changes may demonstrate different results. A common issue for the control group was failure to communicate instructions to the subjects as prescribed in the protocol. This was not as prevalent in the test group which may indicate the added simulator practice impacted the student's performance even though it did not explicitly indicate or model this behavior. Note, the observed issues were not modeled in the simulation, and thus represents potential areas of future improvement.

\section{FUTURE DIRECTIONS}

Testing indicated that the HSS simulator can be an effective audiometry teaching tool, but some potential areas of improvement were identified. The most significant was the need to convert it to a web-based platform for broader availability and accessibility. While remote students can use the present simulator, it would require them to download and install the tool locally. A web-based option would make using the simulator easier, with the only requirement being a web browser.

Recommendations from students using the system included that a video tutorial should be created to either replace or supplement the current text-based instructions. They also 
suggested adding a learning mode, where context-sensitive feedback could be displayed on command during the training phase, but hidden when in an evaluation phase. As a consequence of the test, it was determined that the simulator did not verify the student's compliance with the auditory testing. While the system counts the number of frequency tests that were completed, it does not track if the specified frequencies (1000, 2000, and $4000 \mathrm{~Hz}$ tones) were tested, nor whether there was any repetition of those tests, the number of positive responses indicated, or the sound level used. So while the simulator did provide an assessment of the student's ability to determine the correct result, it did not fully capture the performance as specified in the audiology screening guidelines [1]. These factors were used in the evaluator's grading of the students in the subsequent evaluation phase of the exercise. These are features that will be added to future versions of the simulator.

In terms of research directions, the simulator was tested in this study as a learning supplement to in-person audiology screening education and testing. Future work should assess how a low-cost yet effective simulation tool (compared to high-cost in-person training and testing) could replace in-person training components and perhaps be used as an actual screening assessment tool. The DSR presented in this study provides a foundational framework for achieving these future objectives.

\section{REFERENCES}

[1] American Speech-Language-Hearing Association, "Guidelines for audiologic screening”, 1997.

[2] R. P. Cant, and S. J. Cooper, "Simulation-based learning in nurse education: systematic review.Journal of Advanced Nursing, vol. 66, pp. 3 $-15,2010$.

[3] V. Guise, M. Chambers, and M. Välimäki, "What can virtual patient simulation offer mental health nursing education?", Journal of psychiatric and mental health nursing, vol. 19, no. 5, pp. 410-418, 2012.

[4] C. La Cerra, A. Dante, V. Caponnetto, I. Franconi, E. Gaxhja, C. Petrucci, et al., "Effects of high-fidelity simulation based on life-threatening clinical condition scenarios on learning outcomes of undergraduate and postgraduate nursing students: a systematic review and meta-analysis", BMJ Open, vol. 9, No. 2, e025306, 2019.

[5] R. J. Sherwood, and G. Francis, G., "The effect of mannequin fidelity on the achievement of learning outcomes for nursing, midwifery and allied healthcare practitioners: systematic review and meta-analysis", Nurse education today, vol. 69, pp. 81-94, 2018.

[6] D. K. Brown, "Simulation before clinical practice: the educational advantages”, Audiology Today, vol. 29, no. 5, pp.16-24, 2017.

[7] D. Bakhos, J. Galvin, J. M. Aoustin, M. Robier, S. Kerneis, G. Bechet, ... \& Aussedat, C. (2020). "Training outcomes for audiology students using virtual reality or traditional training methods”, PloS one, vol. 15, no. 12, 2020.

[8] ASHA, "2014 standards for the certificate of clinical competence in speech-language pathology", Council for Clinical Certification in Audiology and Speech-Language Pathology of the American SpeechLanguage-Hearing Association., 2016, retrieved [6/1/2021] from https://www.asha.org/Certification/2014-Speech-LanguagePathology-Certification-Standards.

[9] R. I. Zraick, "Review of the use of standardized patients in speechlanguage pathology clinical education”, International Journal of Therapy and Rehabilitation, vol. 19, no. 2, pp. 112-118, 2012.

[10] C. Nye, S. H. Campbell, S. H. Hebert, C. Short, and M. Thomas", Simulation in advanced practice nursing programs: A North-American survey”, Clinical Simulation in Nursing, vol. 26, no. 3-10, 2019.

[11] W. A. Kaf, C. G. Masterson, N. Dion, S. L. Berg, and M. K. Abdelhakiem, M. K., "Optimizing otoscopy competency in audiology students through supplementary otoscopy training”, Journal of the American Academy of Audiology, vol. 24, no. 9, pp. 859-866, 2013.

[12] A. A. Alanazi, "The use of simulation in audiology education to improve students' professional competency", ProQuest LLC, 2017.

[13] A. Reed, A. Andre, S. Ananthakrishnan, and P. Korczak, "Effectiveness of simulation training on graduate audiology students' auditory brainstem response testing skills”, American Journal of Audiology, pp. 1-10, 2021.

[14] ASHA, "Tests of the middle ear", American Speech-Language-Hearing Association, 2021, retrieved 6/4/2021 from https://www.asha.org/public/hearing/tests-of-the-middle-ear/.

[15] AAP, "Recommendations for preventive pediatric health care. Pediatrics", Committee on Practice and Ambulatory Medicine. American Academy of Pediatrics, vol.105, no.3, pp. 645-646, 2000, retrieved on 6/4/2021 from https://pediatrics.aappublications.org/content/105/3/645.

[16] AAP, "2017 recommendations for preventive pediatric health care”, American Academy of Pediatrics, 2017, retrieved on 6/4/2021 from https://pediatrics.aappublications.org/content/pediatrics/139/4/e2017025 4.full.pdf?embargo=true\&download=true.

[17] CBRN, "Continuing education for license renewal, California Board of Registered Nursing, 2021, retrieved on 6/4/2021 from https://www.rn.ca.gov/licensees/ce-renewal.shtml.

[18] A. S. Niskar, S. M. Kieszak, A. Holmes, E. Esteban, C. Rubin, and D. J. Brody, "Prevalence of hearing loss among children 6 to 19 years of age: the Third National Health and Nutrition Examination Survey. Jama, vol. 279, no. 14, pp. 1071-1075, 1988.

[19] M. J. Guralnick, M. J., "Why early intervention works: A systems perspective”, Infants \& Young Children, vol. 24, pp. 6-28, 2011.

[20] ASHA, "Difference between hearing screening and hearing evaluation", American Speech-Language-Hearing Association, 2021, retrieved 6/4/2021 from https://www.asha.org/public/hearing/hearing-screeningand-hearing-evaluation/.

[21] A. A. Alanazi, M. S. Mohamud, S. S. AlSuwailem, "The effect of simulation learning on audiology and speech-language pathology students' self-confidence related to early hearing detection and intervention: a randomized experiment”, Speech, Language and Hearing, pp. 1-14, 2020

[22] R. Ozelie, P. Panfil, N. Swiderski, and E. Walz, "Hearing voices simulation: impact on occupational therapy students", The Open Journal of Occupational Therapy, vol. 6 no. 4, 10, 2018.

[23] E. Toader, "Clinical simulations for learning medical skills: a work-based approach to simulators", Procedia-Social and Behavioral Sciences, vol. 197, pp. 2443-2448, 2015.

[24] B. M. Halm, M. T. Lee, A. A. Franke, "Improving toxicology knowledge in preclinical medical students using high-fidelity patient simulators". Hawaii Medical Journal, vol. 70, no. 6, pp. 112-115, 2011.

[25] M. M. Muñoz-Montes, I. Villagrán-Gutiérrez, F. Pozo-Tapia, P. TapiaTapia, Y. Castro-Soares, and E. Fuentes-López, "Speech and language therapy students' perception of learning through the incorporation of clinical simulation workshops: a mixed-methods study”, Revista CEFAC, vol. 23, 2021.

[26] R. M. Fanning, and D. M. Gaba, "The role of debriefing in simulationbased learning”, Simulation in Healthcare, vol. 2, no. 2, pp. 115-125, 2007.

[27] S. Gregor and A. R. Hevner, "Positioning and presenting design science research for maximum impact," MIS Quarterly: Management Information Systems, vol. 37, no. 2. University of Minnesota, pp. 337355, 2013, doi: 10.25300/MISQ/2013/37.2.01.

[28] K. Peffers, T. Tuunanen, M. A. Rothenberger, and S. Chatterjee, “A Design Science Research Methodology for Information Systems Research,” J. Manag. Inf. Syst., vol. 24, no. 3, pp. 45-77, 2007, doi: 10.2753/MIS0742-1222240302.

[29] A. Drechsler, "Utilizing, Producing, and Contributing Design Knowledge in DSR Projects," Open Access Victoria University of Wellington | Te Herenga Waka, Jan. 2011. doi: 10.26686/WGTN.13088369.V1. 